\title{
Physicians Perception on Complementary and Alternative Medicine (CAM): A Cross Sectional Survey at Tertiary Care Hospital in India
}

\author{
Nitin S Kunnoor ${ }^{1 *}$, Rajni Rathore ${ }^{2}$ and Denis Xavier $^{2}$
}

${ }^{1}$ Department of Pharmacology, M R Medical College \& Hospital, Kalaburagi, Karnataka, India

${ }^{2}$ Department of Pharmacology, St John's Medical College \& Hospital, Bangalore, India

\begin{abstract}
Background: Allopathic physicians are relatively unfamiliar with Complementary and Alternative Medicine (CAM) but they're in common use. A cross sectional survey was conducted to understand physician's perception of it.

Method: All 385 physicians from St. John's Medical College \& Hospital, Bangalore were approached. Data were collected using anonymised questionnaires on its use, perceptions, recommendation and inclusion in medical curricula. Responses were analysed according to their experience $(<5,5-10 \&>10$ years) and qualifications (specialists with: MD, MS, MCh, DM, Diploma or GPs with MBBS and Fellowship).

Results: Responses were received from 247 (64\%) of physicians. 173/38/36 (70/15/15\%) had <5/5-10/>10 years' experience, half were specialists, half GPs. Their mean age was 33 years, 114 (46\%) were males. 168/169/152/133/91/84 $(68 / 68 / 53 / 54 / 37 / 34 / 27 \%)$ reported their patients used CAM for chronic diseases/consult their patients about CAM/said their patients informed them about it/considered it may be useful but harmful/that it increases side effects of allopathic medication/ that it worsens the condition. 67 (27\%) physicians recommended it. Yoga/meditation, 57/47 (23/19\%) were commonest. Most, $97 / 143 / 154 / 98$ (39/58/62/40\%) disagree with using it when allopathy is ineffective/disagree with including it in medical curricula/disagree on training doctors about it/do not recommend it.
\end{abstract}

Conclusion: CAM is used ubiquitously, especially for chronic diseases. Allopathic doctors need knowledge about them and their appropriate use for better outcomes.

Keywords: Complementary and alternative medicine; Physician; Perception; Chronic diseases

\section{Introduction}

Complementary and Alternative Medicine (CAM) is defined by the National Centre for Complementary and Alternative Medicine (NCCAM) as a group of diverse medical and health care systems, products, and practices that are not presently considered part of conventional medical care, consequently it's not included in the medical curriculum. It is generally assumed that complementary medicine is used in conjunction with allopathic medicine and alternative medicine replaces allopathic medicine [1].

An integrative approach was recently recommended, providing affordable and practical solutions to the global healthcare crisis, especially in developing countries like India. The department of Ayurveda, Yoga and Naturopathy, Unani, Siddha and Homeopathy (AYUSH), under the Ministry of Health and Family Welfare proposed a new approach by integrating with the allopathic system, ensuring health for all citizens across the country [2].

The success of the new, 'integrative', approach depends on its ability to identify the respective values, beliefs, fundamentals, practices, strengths, and weaknesses of all the systems. China's bottom up approach incorporates practices from both traditional and modern medicine effectively. Chinese medical students take compulsory courses in both modern and traditional medicine, applying their knowledge in practice. Therefore, Chinese physicians are aware of the advantages of both systems and can opt for the right combination. The role of integrative medicine is still at its infancy in India [3].

The scope of integration between CAM and allopathic medicine in future depends on the attitudes of allopathic physicians, especially postgraduate residents. Their attitudes and beliefs may strongly affect the way they ultimately practice medicine. In previous studies in developed countries, medical students have consistently expressed interest in gaining more exposure to CAM [4,5]. Medical colleges are becoming aware of the need to provide CAM related education and training [6].

CAM such as Ayurveda, homeopathy, herbal treatment, meditation and yoga are widely used in chronic illnesses such as diabetes mellitus, hypertension, coronary artery disease, stroke, COPD, asthma, arthritis, gout and other chronic neurological diseases. CAM's utilisation in developed and developing countries has almost doubled in recent years, particularly where long term treatment is required [7]. The utilization rate of CAM in India ranges from $32-63 \%$ in chronic medical conditions [8]. Little is known about CAM, particularly their adverse effects on routine allopathic treatments for chronic diseases.

Despite uncertainties about their effectiveness, the use of CAM is well documented. Studies suggest $30-68 \%$ of patients use some form of CAM therapy [9]. Several international studies have surveyed physicians' practices and beliefs on CAM [10]. These studies showed considerable variation in responses regarding physicians' practice, referrals for and belief in the efficacy of specific types of CAM. Possible explanations cited for variations between these surveys include:

(i) Different demographics (greater acceptance of CAM in some European countries);

(ii) Survey wording (including CAM therapy definitions);

${ }^{*}$ Corresponding author: Dr. Nitin S. Kunnoor MBBS; MD. Department of Pharmacology, M R Medical College \& Hospital, Kalaburagi, Karnataka, India, Tel: +91-9964030090; E-mail: drnitinskunnoor@yahoo.in

Received April 15, 2015; Accepted May 06, 2015; Published May 08, 2015

Citation: Kunnoor NS, Rathore R, Xavier D (2015) Physicians Perception on Complementary and Alternative Medicine (CAM): A Cross Sectional Survey at Tertiary Care Hospital in India. Med chem 5: 197-202. doi:10.4172/21610444.1000264

Copyright: (c) 2015 Kunnoor NS, et al. This is an open-access article distributed under the terms of the Creative Commons Attribution License, which permits unrestricted use, distribution, and reproduction in any medium, provided the original author and source are credited. 
(iii) Differences in the ratio of general practitioners to specialists; and

(iv) Local or regional differences in their familiarity or availability [11].

Most allopathic physicians are unaware of CAM's effects in chronic disease. Many studies concern the prevalence, pattern, and frequency of CAM use in various chronic diseases. Few include details of patient's perspectives of CAM. Although studies assessing physicians' perceptions of CAM come primarily from Western countries, data from the developing countries are scarce. Factors influencing Indian views on CAM might be completely different from those prevalent in the West. Routine enquiry of patients' CAM therapies may improve and enhance their care by avoiding potentially harmful drug interactions and the art of medicine by improving the quality of doctor-patient therapeutic relationships. Investing more time on evidence based CAM therapies in medical curriculum may improve communication concerning CAM self-prescribed by many health consumers. Therefore physician's opinion on CAM needs assessment in our specific setting. To broaden their understanding in this area, we conducted a cross sectional survey on their perception on Complementary and Alternative Medicine, gaining important perspectives from treating physicians.

\section{Methods}

This study was approved by the Institutional Ethics Review Board of St. John's Medical College and Hospital, Bangalore. 385 physicians from the departments of Psychiatry, Urology, Dermatology, Paediatrics, Cardiology, Gastroenterology, Neurology, Pain \& palliative, Physical and Medical Rehabilitation, Medical Oncology, Medicine, Surgery, Nephrology, Obstetrics and Gynaecology, Community Medicine, Orthopaedics, Surgical Oncology, Chest Medicine, Emergency Medicine and Endocrinology were approached. Specialities which could not conceivably involve use of alternative medicine in management of chronic diseases, such as radiology and anaesthesiology were excluded.

Data were collected using a structured questionnaire, approaching individual doctors in the hospital during their non-OPD days or during department academic sessions such as seminars, journal clubs or case presentations. Physicians failing to respond after 5 attempts were classed as non-responders. The two page anonymous questionnaire had 14 questions, including details of the prevalence of CAM therapy, opinions on different CAM therapies they know, their use in chronic disease and incorporating CAM education in routine medical curricula. Data on patients' sources of information about CAM, whether they informed the treating doctors about their use of CAM and the physicians' opinion on prescribing CAM or referring patients to CAM therapists were collected.

\section{Data management and statistical analysis}

Data were entered in Epi-Info version 7. Continuous data were analysed using descriptive statistics and categorical data presented as frequencies. Analysis of responses was based on physicians' years of experience (three groups: $<5$ years, 5-10 years and $>10$ years) and qualifications [two groups: Specialist (MD, MS, MCh, DM, Diploma) and General Practitioner (MBBS and Fellowship)]. Groups were compared using Chi-squared tests, p-values $<0.05$ were considered significant. All statistical analyses were performed with SPSS version 17.

\section{Results}

Responses were received from 247 (64\%) of 385 physicians with various years' of experience. Half were specialists, half general physicians. Their median number of years in practice was 2 (IQR; 1-5), mean age 33 years $(\mathrm{SD} \pm 8.6), 114(46 \%)$ were males. Table 1 shows characteristics of responding physicians.

Among 168 (68\%) physicians who reported that their patients use CAM, 104 of them opined that $10-30 \%$ of their patients used CAM for chronic diseases, physicians consult their patients about CAM [169; $(68.4 \%)]$, and said their patients informed them about it [152 (61.1\%)]. 50 physicians reported patients didn't disclose CAM therapy to doctor for personal reasons, 128 that most patients take CAM therapy recommended by friends and relatives.

Half of the physicians considered it may be useful but might cause harm by increasing side effects of allopathic medication, worsening the condition. 67 physicians most commonly recommended yoga or meditation and 22 referred patients to CAM therapies, mostly yoga, meditation and Ayurveda. Senior doctors made most recommendations and referrals. Table 2 shows overall opinion of physicians.

Tables 3 and 4 shows how physicians' years of experiences and specialist experiences influences their recommendations for Ayurveda, homeopathy naturopathy and herbal medicine. Physicians $<10$ years of experience and specialists opined that its patients choice to take CAM therapy. General physicians didn't recommend them. Most physicians were aware of and recommended yoga, meditation, massage, spiritual healing, dance and music therapy but many were unaware of Siddha, Unani, Chiropractic therapy \& Reiki therapy.

Most physicians disapprove CAM therapy for patients refractory to allopathic treatment, including CAM education in medical curricula or training doctors in CAM therapy. Over a third of physicians don't advise their patients to take CAM therapy, see Tables 5 and 6 .

\section{Discussion}

Most respondents to this comprehensive survey of physicians' perceptions on CAM therapies at a tertiary care teaching hospital had $<5$ years of experience. Their mean age was 33 years and median number of years in practice 2. Most physicians reported their patients use CAM for chronic diseases, half opined that CAM may be useful but also harmful. A quarter of physicians recommended CAM to their patients, especially yoga and meditation. A few physicians referred patients to CAM therapies, including Yoga, Meditation and Ayurveda. Senior doctors made most recommendation and referrals.

\begin{tabular}{|l|c|c|}
\hline Age in years & Number & Percentage \\
\hline $20-35$ & 180 & 72.9 \\
\hline $36-45$ & 40 & 89.1 \\
\hline $46-55$ & 21 & 8.5 \\
\hline$>56$ & 6 & 2.4 \\
\hline Gender & & \\
\hline Male & 114 & 46.2 \\
\hline Female & 133 & 53.8 \\
\hline Medical Specializations & & \\
\hline Specialist (DM,MCh,MD,MS,Diploma) 124 & 123 & 51.2 \\
\hline Generalist (MBBS) & & 49.8 \\
\hline Years of experience & 173 & 70.0 \\
\hline$<5$ years & 38 & 15.4 \\
\hline 5-10 years & 36 & 14.6 \\
\hline$>10$ years & & \\
\hline
\end{tabular}

Mean age 33 years $(S D \pm 8.6)$, Mean years since highest degree $5.6(S D \pm 6.1)$ Median number of years of experience 2 (IQR 1-5). IQR-Inter Quartile Range 25\%$75 \%$, SD- Standard Deviation,

Table 1: Characteristics of 247 respondents on CAM. 


\begin{tabular}{|l|c|c|}
\hline Physician opinions on CAM therapy & Number & Percentage \\
\hline Are your patients using CAM for chronic disease & $\mathbf{1 6 8}$ & $\mathbf{6 8 . 0}$ \\
\hline $10-30 \%$ & 104 & 60.8 \\
\hline$<10 \%$ & 43 & 25.1 \\
\hline $31-50 \%$ & 20 & 11.7 \\
\hline >75\% & 3 & 1.8 \\
\hline $51-75 \%$ & 1 & 0.6 \\
\hline Do you ask patients whether they are using CAM & $\mathbf{1 6 9}$ & $\mathbf{6 8 . 4}$ \\
\hline Patients inform you about using CAM & $\mathbf{1 5 1}$ & $\mathbf{5 2 . 6}$ \\
\hline $10-30 \%$ & 71 & 46.7 \\
\hline <10\% & 43 & 28.9 \\
\hline $31-50 \%$ & 18 & 11.8 \\
\hline >75\% & 11 & 7.2 \\
\hline $51-75 \%$ & 8 & 5.3 \\
\hline General utility & & \\
\hline May be useful, but also harmful & 133 & 54.3 \\
\hline Useless, can be harmful & 60 & 24.5 \\
\hline May be useful, but harmless & 16 & 6.5 \\
\hline Don't Know & 14 & 5.7 \\
\hline Is useful and helps in improving patient condition & 13 & 5.3 \\
\hline Useless \& harmless & 9 & 3.7 \\
\hline Interaction with allopathic medication & & \\
\hline Increases side effects of allopathic medicine & 91 & 37.0 \\
\hline Does not affect action of allopathic medicine & 66 & 26.8 \\
\hline No effect, but helps control severity of Disease & 51 & 20.7 \\
\hline Reduces the effect of allopathic medicine & 25 & 10.2 \\
\hline Improves the effect of allopathic medicine & 9 & 3.7 \\
\hline Decreases side effect of allopathic medicine & 4 & 1.6 \\
\hline Effect of CAM & & \\
\hline CAM- Worsen the patient condition & 84 & 34.3 \\
\hline CAM can have mild to moderate side effect & 62 & 25.3 \\
\hline CAM can have serious side effects & 34 & 13.9 \\
\hline CAM-Improves the patient condition & 27 & 11.0 \\
\hline CAM- Doesn't change the patient condition & 24 & 9.8 \\
\hline CAM - Has no side effects & 14 & 5.7 \\
\hline Physicians recommending patients to CAM & $\mathbf{6 7}$ & $\mathbf{2 7 . 1}$ \\
\hline Yoga & 57 & 85.0 \\
\hline Meditation & 47 & 70.1 \\
\hline Massage & 14 & 20.8 \\
\hline Ayurveda & 13 & 19.4 \\
\hline Spiritual healing/prayer & 11.9 \\
\hline Dance/Art/Music therapy & 8.9 \\
\hline Homeopathy & & \\
\hline Acupuncture & & \\
\hline
\end{tabular}

Table 2: Physicians opinion on CAM therapy.

Most physicians opine that CAM shouldn't be used in patients refractory to allopathic treatment and on including CAM education in medical curricula or training doctors on CAM therapies, Most physicians in all groups (based on years of experience and qualification) were aware of and recommended yoga, meditation, massage, spiritual healing, dance and music therapy. They were unaware of Siddha, Unani, Chiropractic therapy \& Reiki therapy.

The $64 \%$ response rate is comparable to that of a nationwide study of New Zealand general practitioners conducted in 2006 [12] and among general practitioners in Doha, Qatar in 2010 [13]. Previous studies have shown utilization of CAM therapy was between 19-68\%, as in our study [14-20].

$68 \%$ of the physicians who responded to our questionnaire said their patients use CAM therapy for chronic disease. A study done in Australia [7] by JCS Mak reported 85\% were familiar with CAM. The commonest therapies were acupuncture (80\%), yoga (74\%) and Tai-Chi $(72 \%)$. We found that majority of physicians, regardless of experience, were familiar with most CAM therapies, recommending yoga and meditation.

About half opined that CAM may be useful but also harmful, over a third said it increases the side effect of allopathic medication and a third thought CAM therapy worsens the condition of patients. Most physicians advise against taking CAM therapy.

A review study on side effects of CAM therapy by Niggemann B and Grubber $\mathrm{C}$ described the adverse effects and drug interactions with

\begin{tabular}{|c|c|c|c|c|c|c|}
\hline $\begin{array}{l}\text { Types of } \\
\text { CAM }\end{array}$ & Experience & Unaware & $\begin{array}{c}\text { Not } \\
\text { recommend }\end{array}$ & Recommend & \begin{tabular}{|l|} 
Patients \\
choice
\end{tabular} & p-valu \\
\hline \multirow{3}{*}{ Ayurveda } & $<5$ & $26(15.1)$ & $51(29.7)$ & 35 (20.3) & $60(34.9)$ & \multirow{3}{*}{0.001} \\
\hline & $5-10$ & $7(18.4)$ & $8(21.1)$ & $7(18.4)$ & $16(42.1)$ & \\
\hline & $>10$ & $17(47.2)$ & $5(13.9)$ & $2(5.6)$ & $12(33.3)$ & \\
\hline \multirow{3}{*}{ Homeopathy } & $<5$ & $27(15.6)$ & $53(30.6)$ & $31(17.9)$ & 61 (35.3) & \multirow{3}{*}{0.003} \\
\hline & $5-10$ & $7(18.4)$ & $8(21.1)$ & $7(18.4)$ & $18(42.1)$ & \\
\hline & $>10$ & $18(50.0)$ & $7(19.4)$ & $3(8.3)$ & $8(22.2)$ & \\
\hline \multirow{3}{*}{ Naturopathy } & $<5$ & $33(19.1)$ & $44(25.4)$ & $40(23.1)$ & $55(31.8)$ & \multirow{3}{*}{0.008} \\
\hline & $5-10$ & $8(21.1)$ & $11(28.9)$ & $7(18.4)$ & $11(28.9)$ & \\
\hline & $>10$ & $18(50.0)$ & $5(13.9)$ & $2(5.6)$ & $11(30.6)$ & \\
\hline \multirow{3}{*}{ Acupuncture } & $<5$ & $28(16.2)$ & $38(22.0)$ & $35(20.2)$ & $71(41.0)$ & \multirow{3}{*}{0.030} \\
\hline & $5-10$ & $11(28.9)$ & $10(26.3)$ & $8(21.1)$ & $8(21.1)$ & \\
\hline & $>10$ & $9(25.0)$ & $2(5.6)$ & $5(13.9)$ & $20(55.6)$ & \\
\hline \multirow{3}{*}{$\begin{array}{l}\text { Herbal } \\
\text { medicine }\end{array}$} & $<5$ & $32(18.5)$ & $57(32.9)$ & $24(13.9)$ & $58(33.5)$ & \multirow{3}{*}{0.006} \\
\hline & $5-10$ & $13(34.2)$ & $8(21.1)$ & $3(7.9)$ & $14(36.8)$ & \\
\hline & $>10$ & $18(50.0)$ & $6(16.70)$ & $1(2.8)$ & $11(30.6)$ & \\
\hline \multirow{3}{*}{ Yoga } & $<5$ & $6(3.5)$ & $4(2.3)$ & $129(74.6)$ & $32(18.5)$ & \multirow{3}{*}{0.285} \\
\hline & $5-10$ & $2(5.3)$ & $2(5.3)$ & $31(81.6)$ & $3(7.9)$ & \\
\hline & $>10$ & $4(11.1)$ & 0 & $24(66.7)$ & $8(22.2)$ & \\
\hline \multirow{3}{*}{ Massage } & $<5$ & $16(9.2)$ & $17(9.8)$ & $81(46.8)$ & $58(33.5)$ & \multirow{3}{*}{0.190} \\
\hline & $5-10$ & $7(18.4)$ & $6(15.8)$ & $20(52.6)$ & $5(13.2)$ & \\
\hline & $>10$ & $6(16.7)$ & $2(5.6)$ & $14(38.9)$ & $14(38.9)$ & \\
\hline \multirow{3}{*}{ Meditation } & $<5$ & $8(4.6)$ & $6(3.5)$ & $122(70.5)$ & $35(20.2)$ & \multirow{3}{*}{0.305} \\
\hline & $5-10$ & $3(7.9)$ & $2(5.3)$ & $27(71.1)$ & $5(13.2)$ & \\
\hline & $>10$ & $5(13.9)$ & 0 & $21(58.3)$ & $10(27.8)$ & \\
\hline \multirow{3}{*}{ Siddha } & $<5$ & $71(41.0)$ & $62(35.8)$ & $6(3.5)$ & $33(19.1)$ & \multirow{3}{*}{0.482} \\
\hline & $5-10$ & $17(44.7)$ & $14(36.8)$ & 0 & $7(18.4)$ & \\
\hline & $>10$ & $22(61.1)$ & $8(22.2)$ & 0 & $6(16.7)$ & \\
\hline \multirow{3}{*}{ Unani } & $<5$ & $76(43.9)$ & $60(34.7)$ & $5(2.9)$ & $31(17.9)$ & \multirow{3}{*}{0.535} \\
\hline & $5-10$ & $17(44.7)$ & $14(36.8)$ & 0 & $7(18.4)$ & \\
\hline & $>10$ & $23(63.9)$ & $8(22.2)$ & 0 & $5(13.5)$ & \\
\hline \multirow{3}{*}{$\begin{array}{c}\text { Spiritual } \\
\text { healing/ } \\
\text { prayer }\end{array}$} & $<5$ & $13(7.5)$ & $13(7.5)$ & $80(46.2)$ & 66 (38.2) & \multirow{3}{*}{0.755} \\
\hline & $5-10$ & $5(13.2)$ & $2(5.3)$ & $20(52.6)$ & $11(28.9)$ & \\
\hline & $>10$ & $6(16.7)$ & $2(5.6)$ & $16(44.4)$ & $12(33.3)$ & \\
\hline \multirow{3}{*}{$\begin{array}{c}\text { Dance, Art, } \\
\text { Music } \\
\text { therapy }\end{array}$} & $<5$ & $14(8.1)$ & $13(7.5)$ & $91(52.6)$ & $54(31.2)$ & \multirow{3}{*}{0.377} \\
\hline & $5-10$ & $4(10.5)$ & $2(5.3)$ & $25(65.8)$ & $7(18.4)$ & \\
\hline & $>10$ & $6(16.7)$ & 0 & $18(50)$ & $12(33.3)$ & \\
\hline & $<5$ & $80(46.2)$ & $33(19.1)$ & $20(11.6)$ & $38(22.0)$ & \\
\hline $\begin{array}{c}\text { Chiropractice } \\
\text { therapy }\end{array}$ & $5-10$ & $20(52.6)$ & $4(10.5)$ & $5(13.2)$ & $9(23.7)$ & 0.696 \\
\hline & $>10$ & $19(52.8)$ & $6(16.7)$ & $1(2.8)$ & $10(27.8)$ & \\
\hline Reiki / & $<5$ & $74(42.8)$ & $39(22.5)$ & $14(8.1)$ & $44(25.4)$ & \\
\hline Therapeutic & $5-10$ & $17(44.7)$ & $5(13.2)$ & $5(13.2)$ & $10(26.3)$ & 0.557 \\
\hline & $>10$ & $20(55.6)$ & $5(13.9)$ & $1(2.8)$ & $10(27.8)$ & \\
\hline
\end{tabular}

Table 3: Physicians responses on different type of CAM therapy based on experience (years), \# (\%). 


\begin{tabular}{|c|c|c|c|c|c|c|}
\hline Types of CAM & Physicians & Unaware & Not recommend & Recommend & Patients choice & $p$-value \\
\hline \multirow{2}{*}{ Ayurveda } & Specialist & $32(25.8)$ & $25(20.2)$ & $16(12.9)$ & $51(41.1)$ & \multirow{2}{*}{0.006} \\
\hline & Gen. physician & $18(14.6)$ & 39 (31.7) & $28(22.8)$ & $37(30.1)$ & \\
\hline \multirow{2}{*}{ Siddha } & Specialist & $58(46.8)$ & 39 (31.5) & $1(0.8)$ & $26(21.0)$ & \multirow{2}{*}{0.267} \\
\hline & Gen. physician & $52(42.3)$ & $45(36.6)$ & $5(4.1)$ & $20(16.3)$ & \\
\hline \multirow{2}{*}{ Unani } & Specialist & $62(50.0)$ & $36(29.0)$ & $2(1.6)$ & 24 (19.4) & \multirow{2}{*}{0.471} \\
\hline & Gen. physician & $54(43.9)$ & $46(37.4)$ & $3(2.4)$ & $19(15.4)$ & \\
\hline \multirow{2}{*}{ Homeopathy } & Specialist & $33(26.6)$ & $30(24.2)$ & $15(12.1)$ & $46(37.1)$ & \multirow{2}{*}{0.056} \\
\hline & Gen. physician & 19 (15.4) & $38(30.9)$ & $26(21.1)$ & $39(31.7)$ & \\
\hline \multirow{2}{*}{ Naturopathy } & Specialist & $35(28.2)$ & $26(21.0)$ & $17(13.7)$ & $45(36.3)$ & \multirow{2}{*}{0.042} \\
\hline & Gen. physician & $24(19.5)$ & $34(27.6)$ & $32(26)$ & $32(26)$ & \\
\hline \multirow{2}{*}{ Herbal medicine } & Specialist & $37(29.8)$ & $30(24.2)$ & $9(7.3)$ & $48(38.7)$ & \multirow{2}{*}{0.024} \\
\hline & Gen. physician & $26(21.1)$ & $41(33.3)$ & $19(15.4)$ & $35(28.5)$ & \\
\hline \multirow{2}{*}{ Acupuncture } & Specialist & $30(24.2)$ & $19(15.3)$ & $22(17.7)$ & $52(41.9)$ & \multirow{2}{*}{0.167} \\
\hline & Gen. physician & $18(14.6)$ & $31(25.2)$ & $26(21.1)$ & $47(38.2)$ & \\
\hline \multirow{2}{*}{ Meditation } & Specialist & $13(10.5)$ & $3(2.4)$ & $82(66.1)$ & $25(20.2)$ & \multirow{2}{*}{0.121} \\
\hline & Gen. physician & $3(2.4)$ & $5(4.1)$ & $88(71.5)$ & $25(20.3)$ & \\
\hline \multirow{2}{*}{ Yoga } & Specialist & $10(8.1)$ & $2(1.6)$ & $88(71.0)$ & $23(18.5)$ & \multirow{2}{*}{0.161} \\
\hline & Gen. physician & $2(1.6)$ & $4(3.3)$ & $96(78.0)$ & $20(16.3)$ & \\
\hline \multirow{2}{*}{ Massage } & Specialist & $19(15.3)$ & $10(8.1)$ & $57(46.0)$ & $38(30.6)$ & \multirow{2}{*}{0.307} \\
\hline & Gen. physician & $10(8.1)$ & $15(12.2)$ & $58(47.2)$ & $39(31.7)$ & \\
\hline \multirow{2}{*}{ Spiritual healing/ prayer } & Specialist & $17(13.7)$ & $8(6.5)$ & $56(45.2)$ & $43(34.7)$ & \multirow{2}{*}{0.243} \\
\hline & Gen. physician & $7(5.7)$ & $9(7.3)$ & $60(48.8)$ & $46(37.4)$ & \\
\hline \multirow{2}{*}{$\begin{array}{c}\text { Dance / Art } \\
\text { Music therapy }\end{array}$} & Specialist & $14(11.3)$ & $3(2.4)$ & $65(52.4)$ & $42(33.9)$ & \multirow{2}{*}{0.065} \\
\hline & Gen. physician & $10(8.1)$ & $12(9.8)$ & $69(56.1)$ & $31(25.2)$ & \\
\hline \multirow{2}{*}{$\begin{array}{l}\text { Chiropractice } \\
\text { therapy }\end{array}$} & Specialist & $57(46)$ & $19(15.3)$ & $11(8.9)$ & $37(29.8)$ & \multirow{2}{*}{0.076} \\
\hline & Gen. physician & $62(50.4)$ & $24(19.5)$ & $15(12.2)$ & $20(16.3)$ & \\
\hline \multirow{2}{*}{ Reiki/Therapeutic touch } & Specialist & $53(42.7)$ & $22(17.7)$ & $8(6.5)$ & $40(32.3)$ & \multirow{2}{*}{0.209} \\
\hline & Gen. physician & $58(47.2)$ & $27(22.0)$ & $12(9.8)$ & $24(19.5)$ & \\
\hline
\end{tabular}

Specialist- MD, MS, MCh, DM, Diploma and General physicians (MBBS and Fellowship)

Table 4: Physicians responses on different type of CAM therapy based on Specilization, \# (\%).

\begin{tabular}{|c|c|c|c|c|c|}
\hline Physicians' Opinion & Group & Disagree & Unsure & Agree & p-value \\
\hline \multirow{5}{*}{ CAM for uncured patients } & $<5$ years & $70(40.5)$ & $68(39.3)$ & $35(20.0)$ & \multirow{3}{*}{0.489} \\
\hline & 5-10 Years & $11(28.9)$ & $15(39.5)$ & $12(33.3)$ & \\
\hline & $>10$ years & $16(44.4)$ & $12(31.6)$ & $8(22.2)$ & \\
\hline & Specialists & $45(46.4)$ & $49(51.6)$ & $30(54.5)$ & \multirow{2}{*}{0.591} \\
\hline & Gen. physicians & $52(53.6)$ & $46(48.4)$ & $25(45.5)$ & \\
\hline \multirow{5}{*}{ CAM in medical curriculum } & $<5$ years & $104(60.1)$ & $25(14.5)$ & $44(25.4)$ & \multirow{3}{*}{0.162} \\
\hline & 5-10Years & $16(42.2)$ & $11(28.9)$ & $11(28.9)$ & \\
\hline & $>10$ years & $23(63.9)$ & $6(16.7)$ & $7(19.5)$ & \\
\hline & Specialists & $66(46.2)$ & $26(61.9)$ & $32(51.6)$ & \multirow{2}{*}{0.193} \\
\hline & Gen. physicians & $77(53.8)$ & $16(38.1)$ & $30(48.4)$ & \\
\hline \multirow{5}{*}{ All doctors need to learn CAM } & $<5$ years & $111(64.2)$ & $34(19.7)$ & $28(16.2)$ & \multirow{3}{*}{0.423} \\
\hline & $5-10$ years & $20(52.6)$ & $10(26.3)$ & $8(21.1)$ & \\
\hline & $>10$ years & $23(63.9)$ & $10(27.8)$ & $3(8.3)$ & \\
\hline & Specialists & $78(50.6)$ & $31(57.4)$ & $15(38.5)$ & \multirow{2}{*}{0.194} \\
\hline & Gen. physicians & $76(49.4)$ & $23(42.6)$ & $24(61.5)$ & \\
\hline
\end{tabular}

Table 5: Physicians opinion on CAM based on years of experience and specializations.

allopathic medication [21]. Most side effects were from acupuncture leading to pneumothorax, cardiac tamponade or spinal injury. Infectious complications after acupuncture include hepatitis and bacterial endocarditis. Organ toxicity has been associated with various herbal preparations involving the liver, kidneys, and the heart. Some herbs may have carcinogenic properties. Severe nutritional deficiencies can occur in infants and small children given strict alternative diets adulterated with steroids [21].

A quarter of our physicians recommended CAM to their patients, most commonly yoga and meditation.
Siddha, Unani, herbal medicine and Reiki/therapeutic touch were rarely recommended. A small proportion willingly referred their patients to CAM practitioners, notably Ayurveda and massage. In contrast a study by JCS Mak in Australia reported $84 \%$ of physicians referred their patients to CAM therapist, $38 \%$ personally used CAM and $94 \%$ inquired about CAM therapies [7].

Our physicians with $<5$ years' experience were more willing to refer patients, agreeing with a recent review by Kurtz et al. [22] However, a review study with 19 surveys conducted between 1982 and 1995 found no differences in referral rates for CAM by age [20-28]. Two surveys 


\begin{tabular}{|c|c|c|c|c|c|}
\hline & Physicians' Opinion & Refuse CAM & Take at Own risk & Free Choice & Advocated CAM \\
\hline \multirow{4}{*}{ Years of experience } & $<5$ years & $68(39.3)$ & $40(23.1)$ & 59 34.1) & $4(2.3)$ \\
\hline & 5-10Years & $13(34.2)$ & $7(18.4)$ & $15(39.5)$ & $1(2.6)$ \\
\hline & $>10$ years & $17(47.2)$ & $6(16.7)$ & $10(27.8)$ & $2(5.6)$ \\
\hline & p value & 0.512 & 0.612 & 0.569 & 0.564 \\
\hline \multirow{3}{*}{ Specialization } & Specialists & $49(50.0)$ & $27(50.9)$ & $41(48.8)$ & $5(71.4)$ \\
\hline & Gen. Physicians & $49(50.0)$ & $26(49.1)$ & $43(51.2)$ & $2(28.6)$ \\
\hline & p-value & 0.959 & 0.903 & 0.753 & 0.255 \\
\hline
\end{tabular}

Table 6: Physicians advice on using CAM based on years of experience and specializations.

$[22,23]$ found referral rates for CAM were higher among female physicians and three found no difference in referral rates according to physician's gender [22-24].

We found most physicians (58\%) disapprove including CAM education in medical curricula, whereas most published surveys indicate physicians wish to learn about most common CAM therapies, a high proportion (62-81\%) agreeing to receive more education on them [25-27]

We report $60 \%$ oppose training allopathy physicians about CAM, unsurprising, since many of them respondents were at medical school and residency during which CAM wasn't widely discussed and rarely included in medical school curricula [28]. Most physicians disapprove CAM for patients refractory to allopathic treatment. 64\% of USA medical schools incorporate some element of CAM to their curricula and students voiced interest in evidence-based CAM [29]. Students need to experience CAM practice and educators should use evidencebased strategies to identify useful interventions, aiming to teach doctors to understand and practice two medical systems appropriately [30].

Dietary supplements like selenium, silver, zinc, fluorine etc which are considered complimentary medicine have beneficial effects in various disease conditions. Selenium supplements protect cattle from hypertension and prevent heart failure in pigs. Silver deficiency may account for cancers $[31,32]$.

In Alzheimer's disease (AD), fluorine disrupts protein folding. The presence of fluorine in tea and fluorine released from breakdown of fluorinated anaesthetic (excreted by the kidneys as aluminum fluoride) gives temporary symptomatic relief from dementia in patients with $\mathrm{AD}$ [33-35]. But supplementing human diets with selenium, silver and fluorine to prevent heart disease, cancers and $\mathrm{AD}$ has yet to be widely accepted.

We had a good response rate to a comprehensive survey covering physician's difficulties in perceiving all possible types of CAM therap. However, it only reports the perceptions of CAM and its use as a therapy throughout one tertiary care hospital in south India treating chronic diseases. Further this study does not provide the views of the doctors from primary care centre.

\section{Conclusion}

Despite the high utilization of CAM therapy by patients, physicians opined that it shouldn't be used to treat chronic diseases and disapprove including CAM education in medical curricula or training doctors on CAM therapy.

CAM isn't all gospel truth, but its use is ubiquitous, especially for chronic diseases. Patients need open advice regarding CAM therapies and allopathic doctors need to be more aware of CAM to utilize evidence based information on better patient outcomes. Further comprehensive studies at different levels of health care are needed to better understand CAM utilization in chronic conditions.

\section{Acknowledgements}

We gratefully thank Christy Vijay (MBBS intern) and Dr. Fernandes Regan respectively for help in data collection and entry. We thank Mrs. Mary Sudeepa and Mr. Pushparaj for coding and labelling the CRFs.

\section{References}

1. WHO (2011) Global status report on non-communicable diseases (NCDs) launched.

2. Singh B, Kumar M, Singh A (2013) Evaluation of implementation status of national policy on Indian systems of medicine and homeopathy 2002: Stakeholders' perspective. Anc Sci Life 33: 103-108.

3. Bodeker G (2001) Lessons on integration from the developing world's experience. BMJ 322: 164-167.

4. Munstedt $\mathrm{K}$, Harren $\mathrm{H}$, von GR, Hackethal A (2011) Complementary and Alternative Medicine: Comparison of Current Knowledge, Attitudes and Interest among German Medical Students and Doctors. Evid Based Complement Alternat Med 790951.

5. Greiner KA, Murray JL, Kallail KJ (2000) Medical student interest in alternative medicine. J Altern Complement Med 6: 231-234.

6. Astin JA, Soeken K, Sierpina VS, Clarridge BR (2006) Barriers to the integration of psychosocial factors in medicine: results of a national survey of physicians. J Am Board Fam Med 19: 557-565.

7. Mak JC, Mak LY, Shen Q, Faux S (2009) Perceptions and attitudes of rehabilitation medicine physicians on complementary and alternative medicine in Australia. Intern Med J 39: 164-169.

8. Mehta DH, Phillips RS, Davis RB, McCarthy EP (2007) Use of complementary and alternative therapies by Asian Americans. Results from the National Health Interview Survey. J Gen Intern Med 22: 762-767.

9. Wahner-Roedler DL, Elkin PL, Lee MC, Vincent A, Gay RE, et al. (2004) Complementary and alternative medicine: use by patients seen indifferent specialty areas in a tertiary-care centre. Evid Based Integr Med 1: 253-260.

10. Wahner-Roedler DL, Vincent A, Elkin PL, Loehrer LL, Gay RE, et al. (2006) Physicians' attitudes toward complementary and alternative medicine and their knowledge of specific therapies: a survey at an academic medical center. Evid Based Complement Alternat Med 3: 495-501.

11. Astin JA, Marie A, Pelletier KR, Hansen E, Haskell WL (1998) A review of the incorporation of complementary and alternative medicine by mainstream physicians. Arch Intern Med 158: 2303-2310.

12. Poynton L, Dowell A, Dew K, Egan T (2006) General practitioners' attitudes toward (and use of) complementary and alternative medicine: a New Zealand nationwide survey. N Z Med J 119: U2361.

13. Al SI, Ismail MF, Yousuf WA, Salama RE (2010) Knowledge, attitudes and practice of general practitioners towards complementary and alternative medicine in Doha, Qatar. East Mediterr Health J 16: 522-527.

14. Bauer BA1 (2000) Herbal therapy: what a clinician needs to know to counsel patients effectively. Mayo Clin Proc 75: 835-841.

15. Jose VM, Bhalla A, Sharma N, Hota D, Sivaprasad S, et al. (2007) Study of association between use of complementary and alternative medicine and noncompliance with modern medicine in patients presenting to the emergency department. J Postgrad Med 53: 96-101.

16. Lee GB, Charn TC, Chew ZH, Ng TP (2004) Complementary and alternative medicine use in patients with chronic diseases in primary care is associated with perceived quality of care and cultural beliefs. Fam Pract 21: 654-660.

17. Chandrashekara S, Anilkumar T, Jamuna S (2002) Complementary and alternative drug therapy in arthritis. J Assoc Physicians India 50: 225-227. 
Citation: Kunnoor NS, Rathore R, Xavier D (2015) Physicians Perception on Complementary and Alternative Medicine (CAM): A Cross Sectional Survey at Tertiary Care Hospital in India. Med chem 5: 197-202. doi:10.4172/2161-0444.1000264

18. Zaman T, Agarwal S, Handa R (2007) Complementary and alternative medicine use in rheumatoid arthritis: an audit of patients visiting a tertiary care centre. Natl Med J India 20: 236-239.

19. Mehrotra R, Bajaj S, Kumar D (2004) Use of complementary and alternative medicine by patients with diabetes mellitus. Natl Med J India 17: 243-245.

20. Kumar D, Bajaj S, Mehrotra R (2006) Knowledge, attitude and practice of complementary and alternative medicines for diabetes. Public Health 120: 705711.

21. Niggemann B, Gruber C (2003) Side-effects of complementary and alternative medicine. Allergy 58: 707-716.

22. Kurtz ME, Nolan RB, Rittinger WJ (2003) Primary care physicians' attitudes and practices regarding complementary and alternative medicine. J Am Osteopath Assoc 103: 597-602.

23. Astin JA, Marie A, Pelletier KR, Hansen E, Haskell WL (1998) A review of the incorporation of complementary and alternative medicine by mainstream physicians. Arch Intern Med 158: 2303-2310.

24. Schachter L, Weingarten MA, Kahan EE (1993) Attitudes of family physicians to nonconventional therapies. A challenge to science as the basis of therapeutics. Arch Fam Med 2: 1268-1270.

25. Milden SP, Stokols D (2004) Physicians' attitudes and practices regarding complementary and alternative medicine. Behav Med 30: 73-82.

26. Levine SM, Weber-Levine ML, Mayberry RM (2003) Complementary and alternative medical practices: training, experience, and attitudes of a primary care medical school faculty. J Am Board Fam Pract 16: 318-326.
27. Hall J, Bulik R, Sierpina V (2003) Community preceptors' attitudes toward and practices of complementary and alternative medicine: a Texas survey. Tex Med 99: 50-53.

28. Eisenberg DM, Kessler RC, Foster C, Norlock FE, Calkins DR, et al. (1993) Unconventional medicine in the United States. Prevalence, costs, and patterns of use. N Engl J Med 328: 246-252.

29. Wetzel MS, Eisenberg DM, Kaptchuk TJ (1998) Courses involving complementary and alternative medicine at US medical schools. JAMA 280: 784-787.

30. Shim BS, Koh BH, Ahn KS (2004) Education in Oriental Medicine in Kyung Hee University. Evid Based Complement Alternat Med 1: 331-334.

31. Smolders G (2012) Effect of vitamin E and selenium and different types of milk on health and growth of organic goat kids. Tackling the Future Challenges of Organic Animal Husbandry.

32. Nielsen TK, Wolstrup C, Schirmer AL, Jensen PT (1989) Mulberry heart disease in young pigs without vitamin $\mathrm{E}$ and selenium deficiency. Vet Rec 124: 535-537.

33. Strauss ME, Pasupathi M, Chatterjee A (1993) Concordance between observers in descriptions of personality change in Alzheimer's disease. Psychol Aging 8: 475-480.

34. Walker MC, Wen M, Weeks AM, Chang MC (2012) Temporal and fluoride control of secondary metabolism regulates cellular organofluorine biosynthesis. ACS Chem Biol 7: 1576-1585.

35. Michael T Deans. The biochemistry of fluorine, Alzheimer's Disease and air pollution. 